Research Paper

\title{
Association study of polymorphisms in miRNAs with T2DM in Chinese population
}

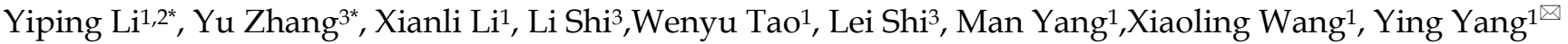 \\ and Yufeng $\mathrm{Yao}^{3 凶}$ \\ 1. Department of Endocrinology and Metabolism, The Second People's Hospital of Yunnan Province \& The Fourth Affiliated Hospital of Kunming Medical \\ University, Kunming 650021, Yunnan, China \\ 2. Key Laboratory of Fertility Regulation and Eugenics of Minority Research of Yunnan Province, Kunming 650091, Yunnan, China \\ 3. Institute of Medical Biology, Chinese Academy of Medical Sciences \& Peking Union Medical College, Kunming 650118, Yunnan, China \\ *These authors contributed equally to this work. \\ $\square$ Corresponding authors: Prof. Ying Yang, Department of Endocrinology and Metabolism, The Second People's Hospital of Yunnan Province, \\ Kunming 650021, Yunnan, China. Email address: yangying2072@126.com. Dr. Yufeng Yao, Institute of Medical Biology, Chinese Academy of Medical \\ Sciences \& Peking Union Medical College, Kunming 650118, Yunnan, China, Email address: leoyyf@gmail.com or yufeng_yao@imbcams.com.cn
}

() 2015 Ivyspring International Publisher. Reproduction is permitted for personal, noncommercial use, provided that the article is in whole, unmodified, and properly cited. See http://ivyspring.com/terms for terms and conditions.

Received: 2015.06.14; Accepted: 2015.09.20; Published: 2015.10.20

\begin{abstract}
Accumulated evidence indicates that microRNA (miRNA or miR) is involved in the development of type 2 diabetes (T2DM). Several studies have shown that single nucleotide polymorphisms (SNPs) located in miRNAs are associated with T2DM in Caucasian populations. The association studies of miRNA's SNPs with T2DM in Asian are rarely reported, and there are distinct genetic differences between Caucasian and Asian populations. The focus of this study, therefore, is the association of T2DM with five SNPs (rs895819 in miR-27a, rs531564 in miR-124a, rs 11888095 in miR-128a, rs3820455 in miR-194a and rs2910164 in miR-146a) located in five miRNAs in a Han Chinese population. A total of 738 subjects with T2DM and 610 non-diabetic subjects were genotyped using the TaqMan method. Next, the associations between the five SNPs with T2DM and individual metabolic traits were evaluated. Our data showed that the $C$ allele of rs531564 in miR-124a may protect against T2DM $(P=0.009, O R=0.758 ; 95 \% C l: 0.616-0.933)$. Conversely, the $C$ allele of rs2910164 in miR-146a may increase the risk of developing T2DM $(P<0.001, O R=1.459$; 95\%Cl: 1.244-1.712). However, these five SNPs did not exhibit significant associations with individual metabolic traits in either the T2DM or non-diabetic groups. Our results revealed that genetic variations in miRNAs were associated with T2DM susceptibility in a Han Chinese population, and these results highlight the need to study the functional effects of these variants in miRNAs on the risk of developing T2DM.
\end{abstract}

Key words: Polymorphisms; T2DM; Chinese population; MicroRNA

\section{Introduction}

Diabetes threatens to overwhelm the health-care system in China. In 2011, the prevalence of all types of diabetes in China rose to a staggering 11.6\%[1]. Type 2 diabetes mellitus (T2DM), which accounts for $85-95 \%$ of all diabetes cases, is characterized by complex traits and may result from both genetic and environmental factors.

Recently, genome-wide association studies have proven a considerable number of single nucleotide polymorphisms (SNPs) to be the basis of the genetic risk factors for T2DM, such as the SNPs in TCF7L2,
PPARY and KCNJ11[2-8]. However, these SNPs can only explain approximately $10 \%$ of the heritability of T2DM $[9,10]$. Until recently, few studies have focused on examining the factors involved in the regulation of gene transcription and translation, such as microRNA (miRNA or miR).

miRNA, which is generated from endogenous hairpin structured transcripts throughout the genome, is estimated to regulate at least $20-30 \%$ of all human genes[11,12]. miRNAs are small, single-stranded molecules with lengths of 21-23 nucleo- 
tides that serve as posttranscriptional regulators of mRNA. By binding to miRNA recognition elements in the 3'untranslated region of mRNA, miRNA promotes mRNA degradation, inhibits its translation, negatively regulates target gene expression[12,13], changes protein expressions and causes disease[14].

Recently, a number of studies have reported that miRNA contributes to the development of T2DM[15-19]. For example, miR-124a, abundant in the pancreatic beta cells, could regulate not only pancreatic $\beta$ cell differentiation but also insulin secretion through its target gene, FoxA2 (forkhead box protein A2, also known as HNF3 $\beta$ )[20]. Moreover, both in vitro and in vivo studies have provided evidence that miR-146a plays an important role in the proinflammatory state in diabetes[21]

SNPs located in a miRNA region could affect the expression of target genes and be involved in the pathogenesis of T2DM. In 2013, Ciccacci et al. reported associations between SNPs in miRNAs and T2DM in a Caucasian population[22]. Due to the significant genetic differences between Caucasian and Asian populations, it seemed the important to evaluate the association of five SNPs (rs895819 in miR-27a, rs531564 in miR-124a, rs11888095 in miR-128a, rs3820455 in miR-194a and rs2910164 in miR-146a) in miRNAs with the presence of T2DM in a Chinese population. In addition, we also evaluated the association of these SNPs with individual metabolic traits in both the T2DM and non-diabetic (NDM) groups.

\section{Materials and Methods}

\section{Ethics statement}

This protocol was in accordance with the Declaration of Helsinki and was approved by the Institutional Review Boards of the Second People's Hospital of Yunnan Province. All participants provided written informed consent.

\section{Subjects}

A total of 738 patients (467 males and 271 females) who were diagnosed with T2DM at the Second People's Hospital of Yunnan Province from December 2011 to November2014 were recruited into this study. The diagnosis of T2DM was confirmed using the World Health Organization criteria from 1999. The known duration of T2DM in the study participants ranged from 3 months to 28 years. The NDM group included 610 subjects (355 males and 255 females) with no family history of diabetes mellitus and who were recruited from individuals undergoing routine health checkups at the Second People's Hospital of Yunnan Province. Subjects with diabetes or impaired glucose tolerance were excluded from the NDM group on the basis of an oral glucose tolerance test. In addition, subjects with hypertension or coronary heart disease were also excluded from the study. All participants (T2DM and NDM) self-reported to be ethnically Han.

\section{Laboratory measurements}

Venous blood samples were collected in the morning after the subjects had fasted for 12 hours. Fasting plasma glucose (FPG) was assayed using the glucose oxidase method. Total cholesterol (TC), high-density lipoprotein cholesterol (HDL-C), triglycerides (TG) and low-density lipoprotein cholesterol (LDL-C) were determined by enzymatic methods. Glycosylated hemoglobin (HbA1c) was determined by immunoturbidimetry. All laboratory measurements were performed on a HITACHI 7600-020 Automatic Analyzer.

\section{miRNA SNP genotyping}

Genomic DNA was extracted from peripheral lymphocytes using a standard hydroxybenzene-chloroform method. Five SNPs (rs895819 in miR-27a, rs531564 in miR-124a, rs11888095 in miR-128a, rs3820455 in miR-194a and rs2910164 in miR-146a) in miRNAs were detected by PCR amplification using a TaqMan assay (Applied Biosystems, Foster City, CA, USA). Some of the PCR products were characterized by direct sequencing on a 3100 Genetic Analyzer (Applied Biosystems, Tokyo, Japan) using the BigDye Terminator v3.1 Cycle Sequencing Kit (Applied Biosystems) after purification with Sephadex ${ }^{\mathrm{TM}}$ G-50 (GE Healthcare, Piscataway, NJ, USA).

\section{Statistical analysis}

SNPs were tested for Hardy-Weinberg equilibrium (HWE) in both the T2DM and NDM groups. The allele and genotype frequencies of the SNPs were calculated by the direct-counting method. A $\chi^{2}$ test was used to determine differences in allele and genotype frequencies between the T2DM and NDM groups and the odds ratios (OR) with associated 95\% confidence intervals (CI) of genotype-specific risks. The association between each SNP and T2DM was analyzed for mode of inheritance using SNPStats[23]. The Akaike information criterion (AIC) and Bayes information criterion (BIC) were used to determine the best fit model for each SNP. Analysis of variance (ANOVA) was used to compare the differences in metabolic traits between three genotype groups and these five SNPs. Bonferroni correction was used to compare the differences in metabolic traits between two of the three genotype groups. Statistical analyses were performed using SPSS 13 (Chicago, IL). A $P$ value of less than 0.05 was considered statistically significant. 


\section{Results}

\section{Subject characteristics}

Table 1 lists the characteristics of the enrolled subjects. There were no age or gender differences between the T2DM and NDM groups, although the clinical values for metabolic traits, including FPG, TC, HDL-C, TG, LDL-C and HbA1c were significantly different between T2DM and NDM subjects (Table 1).

Table 1. Clinical characteristics of the subjects enrolled in the present study (Data are mean \pm SD)

\begin{tabular}{llll}
\hline & Type 2 diabetes & $\begin{array}{l}\text { Non-diabetic } \\
\text { subjects }\end{array}$ & $P$ \\
\hline $\mathrm{n}$ & 738 & 610 & \\
Age(years) & $50.592 \pm 11.852$ & $49.931 \pm 11.264$ & 0.295 \\
$\begin{array}{l}\text { Sex(M/F) } \\
\text { Total cholesterol(mmol/L) }\end{array}$ & $4.898 \pm 1.099$ & $4.429 \pm 0.868$ & $<0.001$ \\
$\begin{array}{l}\text { Triglycerides(mmol/L) } \\
\text { High-density lipopro- }\end{array}$ & $2.645 \pm 2.062$ & $1.675 \pm 1.118$ & $<0.001$ \\
tein-cholesterol(mmol/L) & $1.084 \pm 0.278$ & $1.286 \pm 0.288$ & $<0.001$ \\
$\begin{array}{l}\text { Low-density lipopro- } \\
\text { tein-cholesterol(mmol/L) }\end{array}$ & $2.672 \pm 0.985$ & $2.090 \pm 0.617$ & $<0.001$ \\
$\begin{array}{l}\text { Fasting plasma glu- } \\
\text { cose(mmol/L) }\end{array}$ & $7.950 \pm 2.466$ & $4.959 \pm 0.627$ & $<0.001$ \\
HbA1C(\%) & $8.910 \pm 2.691$ & $5.200 \pm 0.502$ & $<0.001$ \\
\hline
\end{tabular}

\section{Association of the five SNPs with T2DM}

The allele and genotype frequencies for five SNPs in miRNA are listed in Table 2. The genotype frequencies for SNPs were in HWE for the T2DM and NDM groups $(P>0.05)$, except for rs895819 in the
T2DM group $(\mathrm{P}=0.022)$. In Table 2 , the allele and genotype distribution of the rs895819 in miR-27a, rs11888095 in miR-128a and rs3820455 in miR-194a showed no association with T2DM (P>0.05). However, the allele and genotype distribution of rs531564 in miR-124a and rs2910164 in miR-146a showed association with T2DM $(\mathrm{P}<0.05)$ (Table 2).

\section{Mode of inheritance analysis}

Tables 3-4 and Supplementary Tables 1-3 present the results of analyses to determine the mode of inheritance for the five SNPs. To compare each inheritance model (codominant, dominant, recessive, overdominant and log-additive) to the most general model (codominant), the AIC and BIC were calculated to identify the inheritance model that best fit the data[20]. The model with the smallest AIC and BIC value corresponds to the minimal expected entropy[20]. The best fit inheritance model with the lowest AIC for rs531564 and rs2910164 was additive. For rs531564, the $(2 \mathrm{CC}+\mathrm{CG})$ genotype was protective against T2DM $(\mathrm{P}=0.011$, OR=0.77; 95\%CI: 0.63-0.94) in the additive inheritance model. The rs2910164 (2GG+CG) genotype was also a protective factor against T2DM $(\mathrm{P}<$ $0.001, \mathrm{OR}=0.70$; $95 \% \mathrm{CI}$ : $0.60-0.82$ ) in the additive inheritance model.

\section{Association between genotype and metabolic traits}

No significant associations between the five SNP genotypes were observed with metabolic traits in both the T2DM and NDM groups (data not shown).

Table 2. Comparison of genotypic and allelic distribution of five SNPs (rs895819, rs531564, rs1 1888095, rs3820455 and rs2910164) between T2DMand NDM

\begin{tabular}{|c|c|c|c|c|c|c|c|c|c|c|c|}
\hline SNPs & Allele & & Chi2 & $P$ & Odds Ratio [95\%CI] & & & & Chi2 & $P$ & H-W \\
\hline rs895819 & $\mathrm{C}$ (freq) & $T$ (freq) & & & & $\mathrm{C} / \mathrm{C}$ (freq) & $\mathrm{C} / \mathrm{T}$ (freq) & $\mathrm{T} / \mathrm{T}$ (freq) & & & \\
\hline T2DM & $412(0.279)$ & $1064(0.721)$ & 0.957 & 0.328 & $1.089[0.918 \sim 1.292]$ & $45(0.061)$ & $322(0.436)$ & $371(0.503)$ & 2.525 & 0.283 & 0.022 \\
\hline NDM & $320(0.262)$ & $900(0.738)$ & & & & $40(0.066)$ & $240(0.393)$ & $330(0.541)$ & & & 0.681 \\
\hline rs531564 & $C$ (freq) & $\mathrm{G}($ freq) & & & & $\mathrm{C} / \mathrm{C}($ freq) & $\mathrm{C} / \mathrm{G}$ (freq) & $\mathrm{G} / \mathrm{G}$ (freq) & & & \\
\hline T2DM & $208(0.141)$ & $1268(0.859)$ & 6.866 & 0.009 & $0.758[0.616 \sim 0.933]$ & $17(0.023)$ & $174(0.236)$ & $547(0.741)$ & 6.992 & 0.030 & 0.476 \\
\hline NDM & $217(0.178)$ & $1003(0.822)$ & & & & $26(0.043)$ & $165(0.270)$ & $419(0.687)$ & & & 0.064 \\
\hline rs11888095 & $\mathrm{C}($ freq $)$ & $\mathrm{T}$ (freq) & & & & $\mathrm{C} / \mathrm{C}$ (freq) & $\mathrm{C} / \mathrm{T}$ (freq) & $\mathrm{T} / \mathrm{T}$ (freq) & & & \\
\hline T2DM & $1219(0.826)$ & $257(0.174)$ & 0.866 & 0.352 & $0.908[0.741 \sim 1.113]$ & $507(0.687)$ & $205(0.278)$ & $26(0.035)$ & 1.120 & 0.571 & 0.353 \\
\hline NDM & $1024(0.839)$ & $196(0.161)$ & & & & $430(0.705)$ & $164(0.269)$ & $16(0.026)$ & & & 0.939 \\
\hline rs3820455 & $\mathrm{C}$ (freq) & $T($ freq) & & & & $\mathrm{C} / \mathrm{C}$ (freq) & $\mathrm{C} / \mathrm{T}$ (freq) & $\mathrm{T} / \mathrm{T}$ (freq) & & & \\
\hline T2DM & $179(0.121)$ & $1297(0.879)$ & 2.809 & 0.094 & $1.231[0.965 \sim 1.570]$ & $10(0.014)$ & $159(0.215)$ & $569(0.771)$ & 3.983 & 0.136 & 0.768 \\
\hline NDM & $123(0.101)$ & 1097(0.899) & & & & $9(0.015)$ & $105(0.172)$ & $496(0.813)$ & & & 0.211 \\
\hline rs2910164 & $\mathrm{C}($ freq $)$ & $G($ freq) & & & & $\mathrm{C} / \mathrm{C}$ (freq) & $\mathrm{C} / \mathrm{G}$ (freq) & G/G(freq) & & & \\
\hline T2DM & $1024(0.694)$ & $452(0.306)$ & 21.644 & $<0.001$ & $1.459[1.244 \sim 1.712]$ & $364(0.493)$ & $296(0.401)$ & $78(0.106)$ & 20.243 & $<0.001$ & 0.128 \\
\hline NDM & $742(0.608)$ & $478(0.392)$ & & & & $236(0.387)$ & $270(0.443)$ & $104(0.170)$ & & & 0.078 \\
\hline
\end{tabular}


Table 3. Different inheritance models analysis of the SNP rs531564 in miR-124a between the T2DM and NDM group

\begin{tabular}{|c|c|c|c|c|c|c|c|}
\hline Model & Genotype & NDM(freq) & T2DM(freq) & Odds Ratio [95\%CI] & $P$ & AIC & BIC \\
\hline \multirow[t]{3}{*}{ Codominant } & $\mathrm{G} / \mathrm{G}$ & $419(0.687)$ & $547(0.741)$ & 1.00 & 0.031 & 1855.6 & 1871.2 \\
\hline & $\mathrm{C} / \mathrm{G}$ & $165(0.271)$ & $174(0.236)$ & $0.81[0.63 \sim 1.04]$ & & & \\
\hline & $\mathrm{C} / \mathrm{C}$ & $26(0.043)$ & $17(0.023)$ & $0.50[0.27 \sim 0.94]$ & & & \\
\hline \multirow{2}{*}{ Dominant } & $\mathrm{G} / \mathrm{G}$ & $419(0.687)$ & $547(0.741)$ & 1.00 & 0.028 & 1855.7 & 1866.1 \\
\hline & $\mathrm{C} / \mathrm{G}-\mathrm{C} / \mathrm{C}$ & $191(0.313)$ & $191(0.259)$ & $0.77[0.60 \sim 0.97]$ & & & \\
\hline \multirow[t]{2}{*}{ Recessive } & $\mathrm{G} / \mathrm{G}-\mathrm{C} / \mathrm{G}$ & $584(0.957)$ & $721(0.977)$ & 1.00 & 0.042 & 1856.4 & 1866.8 \\
\hline & $\mathrm{C} / \mathrm{C}$ & $26(0.043)$ & $17(0.023)$ & $0.53[0.28 \sim 0.99]$ & & & \\
\hline \multirow[t]{2}{*}{ Overdominant } & $\mathrm{G} / \mathrm{G}-\mathrm{C} / \mathrm{C}$ & $445(0.730)$ & $564(0.764)$ & 1.00 & 0.140 & 1858.4 & 1868.8 \\
\hline & $\mathrm{C} / \mathrm{G}$ & $165(0.271)$ & $174(0.236)$ & $0.83[0.65 \sim 1.06]$ & & & \\
\hline Log-additive & --- & --- & --- & $0.77[0.63 \sim 0.94]$ & 0.011 & 1854.1 & 1864.5 \\
\hline
\end{tabular}

Table 4. Different inheritance models analysis of the SNP rs2910164 in miR-146a between the T2DM and NDM group

\begin{tabular}{|c|c|c|c|c|c|c|c|}
\hline Model & Genotype & NDM & T2DM & Odds Ratio $[95 \% \mathrm{CI}]$ & $P$ & AIC & BIC \\
\hline \multirow[t]{3}{*}{ Codominant } & $\mathrm{C} / \mathrm{C}$ & $236(0.387)$ & $364(0.493)$ & 1.00 & $<0.001$ & 1842.3 & 1857.9 \\
\hline & $\mathrm{C} / \mathrm{G}$ & $270(0.443)$ & $296(0.401)$ & $0.71[0.56 \sim 0.90]$ & & & \\
\hline & $\mathrm{G} / \mathrm{G}$ & $104(0.171)$ & $78(0.106)$ & $0.49[0.35 \sim 0.68]$ & & & \\
\hline \multirow[t]{2}{*}{ Dominant } & $\mathrm{C} / \mathrm{C}$ & $236(0.387)$ & $364(0.493)$ & 1.00 & $<0.001$ & 1845.2 & 1855.6 \\
\hline & $\mathrm{C} / \mathrm{G}-\mathrm{G} / \mathrm{G}$ & $374(0.613)$ & $374(0.507)$ & $0.65[0.52 \sim 0.81]$ & & & \\
\hline \multirow[t]{2}{*}{ Recessive } & $\mathrm{C} / \mathrm{C}-\mathrm{C} / \mathrm{G}$ & $506(0.830)$ & $660(0.894)$ & 1.00 & $<0.001$ & 1848.6 & 1859.0 \\
\hline & $\mathrm{G} / \mathrm{G}$ & $104(0.171)$ & $78(0.106)$ & $0.58[0.42 \sim 0.79]$ & & & \\
\hline \multirow[t]{2}{*}{ Overdominant } & $\mathrm{C} / \mathrm{C}-\mathrm{G} / \mathrm{G}$ & $340(0.557)$ & $442(0.599)$ & 1.00 & 0.120 & 1858.2 & 1868.6 \\
\hline & $\mathrm{C} / \mathrm{G}$ & $270(0.443)$ & $296(0.401)$ & $0.84[0.68 \sim 1.05]$ & & & \\
\hline Log-additive & --- & --- & -- & $0.70[0.60 \sim 0.82]$ & $<0.001$ & 1840.3 & 1850.7 \\
\hline
\end{tabular}

\section{Discussion}

The Han Chinese population is more genetically predisposed to T2DM than European populations $[24,25]$. It is therefore imperative to explore the genetic architecture and T2DM susceptibility loci that are unique to the Han Chinese population[26]. In this study, we evaluated the associations between five SNPs in miRNAs with T2DM in a Han Chinese population. Our results showed that among these SNPs, rs531564 in miR-124a and rs2910164 in miR-146a were associated with T2DM. In particular, the C allele for rs531564 was associated with a low risk of T2DM. Conversely, the $\mathrm{C}$ allele for rs2910164 was associated with an increased risk of T2DM. The other three SNPs (rs895819 in miR-27a, rs11888095 in miR-128a and rs3820455 in miR-194a) had no observed associations with T2DM in allele, genotype nor in different heritance models.

The miR-124a has been identified as being involved in the development of the pancreas and the differentiation of pancreatic $\beta$ cells $[20,27]$. Moreover, recent study has shown that miR-124a was hyper expressed in T2DM human pancreatic islet and its overexpression impaired the glucose-stimulated insulin secretion[28]. In 2013, Ciccacci et al. proved rs531564 in miR-124a, which is located in the pri-miR region, was associated with T2DM in an Italian population, and the $\mathrm{G}$ allele of rs531564 was the risk allele of T2DM[22]. In the current study, we also observed that rs531564 in miR-124a was associated with T2DM in a Han Chinese population, and the $\mathrm{C}$ allele of rs531564 was associated with a low risk of T2DM. In 2012, Qi L et al. showed that the G variant allele of rs531564 in miR-124a can alter the stability of the pri-miR and lead to a different mature miRNA structure; also, the amount of mature miR-124a in the CC genotype was $20 \%$ lower than in the GG genotype[29]. Thus, the miR-124a overexpression caused by the G allele could alter insulin secretion and create increased susceptibility to the development of T2DM.

The miR-128a and miR-194a were the predicted miRNAs targeted on the 3' UTR of T2DM-associated genes involved in T2DM susceptibility analyzed by specific software[22]. The T2DM-associated genes are IRS1, INSR, CAPN10, PPARY, TCF7L2 and KCNJ11[2-8]. However, in the current study, we did not observe the association of rs11888095 in miR-128a and rs3820455 in miR-194a with T2DM. Moreover, Ciccacci et al. also failed to observe an association between these two SNPs with T2DM in an Italian population [22]. The reasons for the lack of associations of these two SNPs with T2DM could be miR-128a and miR-194a were predicted using software only, while no studies have directly proved their role in the development of T2DM. In addition, it could also be that these two SNPs might influence neither the structure nor function of those miRNAs.

Recently, miR-27a was found to be highly expressed in adipose tissue, and its suppression may be linked with adipocyte development by repressing PPARY expression[30]. In 2010, Sun Q et al. reported 
that SNP-rs895819 (A/G) within the miR-27a and the AG and GG genotypes of miR-27a increase the amount of miR-27a when compared to the wild-type genotype[31]. In 2013, Ciccacci et al. reported that the G allele of rs895819 in miR-27a had a protective effect against T2DM in an Italian population[22]. However, in the current study, our results showed rs895819 in miR-27a was not associated with T2DM in a Han Chinese population. Interestingly, in 2014, Ciccacci et al. reported that the $\mathrm{G}$ allele of rs895819 in miR-27a was associated with a higher risk of early development of cardiovascular autonomic neuropathy[32]. Ciccacci et al. thought the cause of these different results may be that miR-27a was possibly involved in different pathways in both T2DM and diabetic cardiovascular autonomic neuropathy[32]. In the current study, because we did not collect data concerning complications in the T2DM subject, we do not have any further analysis for the association of these SNPs with complications of T2DM, which may be one of the limitations of our study.

The studies about miR-146a functions indicated

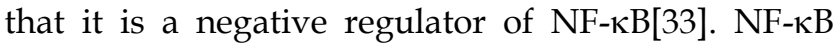
activation promotes insulin resistance and is involved in the pathogenesis of T2DM [34,35]. Moreover, impaired miR-146a levels were observed in diabetic subjects or models[21,36-38]. In the current study, we observed that the rs2910164 C allele in miR-146a may increase the risk of T2DM in a Han Chinese population. In 2008, Jazdzewski $\mathrm{K}$ et al. proved that the rs2910164 C allele could interfere with binding to the nuclear factor of pre-miR-146a, reducing the amount of pre-miR-146a and mature miR-146a[39]. Thus, the rs2910164 C allele with decreased expression of miR-146a may increase susceptibility to T2DM. However, Ciccacci et al. failed to detect the association of rs2910164 with T2DM in an Italian population[22]. The genetic differences between Caucasian and Asian populations may illustrate the different results. Firstly, the $\mathrm{G}$ allele was a minor allele and the frequency was approximately $30-40 \%$ in the Han Chinese population. However, the $\mathrm{C}$ allele is a minor allele with a frequency of $22-26 \%$ in the European population (http:/ / asia.ensembl.org /Homo_sapiens/Variation/ Population? $\mathrm{db}=$ core; $\mathrm{r}=5: 160484911-160485911 ; \mathrm{v}=$ rs2910164; $v d b=v a r i a t i o n ; v f=2579885)$. Secondly, the miR-146a expression level in T2DM subjects showed the discrepancy in different studies[36,40,41]. In 2011, Balasubramanyam et al. reported that the expression level of miR-146a was significantly lower in peripheral blood mononuclear cells in Indian T2DM subjects[36]. Otherwise, circulating miR-146a levels were higher in newly diagnosed T2DM subjects in a Han Chinese population[40,41]. However, Zampetaki et al. showed that there was no notable association between plasma miR-146a and T2DM in an Italian population[42]. Another reason may result from the complexity of miRNA regulation[43]. In other words, each miRNA could have the potential to regulate multiple genes[43], and the same miRNA could be involved in the different pathways in T2DM and its complication, just as miR-27a may play different roles in T2DM and the complications thereof.

\section{Conclusions}

To our best knowledge, this study provided the first evidence regarding the associations of five SNPs located in miR-27a, miR-124a, miR-128a, miR-194a and miR-146a with T2DM in an Asian population. Our study found that rs531564 in the miR-124a C allele may be associated with protective factors against the development of T2DM and that the rs531564 $(2 \mathrm{CC}+\mathrm{CG})$ genotype has a lower risk of T2DM under additive models. Otherwise, the $\mathrm{C}$ allele of rs2910164 in miR-146a might increase the risk of T2DM. Moreover, the rs2910164 (2GG+CG) genotype may have a lower risk of T2DM under additive models. Our results revealed the genetic variation in miRNA might be involved the development of T2DM in the Han Chinese population. These results will provide evidence for the need to study the functional effects of variants miRNA on T2DM. In addition, the genetic differences between Caucasian and Asian populations and the complexity of miRNA regulation in T2DM and in the complications of T2DM may contribute to the unexpected results.

\section{Supplementary Material}

Supplementary tables 1-3.

http://www.medsci.org/v12p0875s1.pdf

\section{Acknowledgments}

This work was supported by grants from the Association Foundation Program of Yunnan Provincial Science and Technology Department and Kunming Medical University (No. 2013FB181), National Natural Science Foundation of China (No. 81260135), Key Laboratory for Fertility Regulation and Eugenics of Minority Research of Yunnan Province (No. ZDSYS2014003).

\section{Competing Interests}

The authors have declared that no competing interest exists.

\section{References}

1. Xu Y, Wang L, He J, et al. Prevalence and control of diabetes in Chinese adults. JAMA. 2013; 310: 948-959.

2. Timpson NJ, Lindgren CM, Weedon $\mathrm{MN}$, et al. Adiposity-related heterogeneity in patterns of type 2 diabetes susceptibility observed in genome-wide association data. Diabetes. 2009; 58: 505-510. 
3. Takeuchi $\mathrm{F}$, Serizawa M, Yamamoto $\mathrm{K}$, et al. Confirmation of multiple risk Loci and genetic impacts by a genome-wide association study of type 2 diabetes in the Japanese population. Diabetes. 2009; 58: 1690-1699.

4. Zeggini E, Weedon MN, Lindgren CM, et al. Replication of genome-wide association signals in UK samples reveals risk loci for type 2 diabetes. Science. 2007; 316: 1336-1341.

5. Scott LJ, Mohlke KL, Bonnycastle LL, et al. A genome-wide association study of type 2 diabetes in Finns detects multiple susceptibility variants. Science. 2007; 316: 1341-1345

6. Sladek R, Rocheleau G, Rung J, et al. A genome-wide association study identifies novel risk loci for type 2 diabetes. Nature. 2007; 445: 881-885.

7. Hara K, Fujita H, Johnson TA, et al. Genome-wide association study identifies three novel loci for type 2 diabetes. Hum Mol Genet. 2014; 23: 239-246.

8. Consortium STD, Williams AL, Jacobs SB, et al. Sequence variants in SLC16A11 are a common risk factor for type 2 diabetes in Mexico. Nature. 2014; 506: 97-101.

9. Ahlqvist E, Ahluwalia TS, Groop L. Genetics of type 2 diabetes. Clin Chem. 2011; 57: 241-254.

10. Bonnefond A, Froguel P, Vaxillaire M. The emerging genetics of type 2 diabetes. Trends Mol Med. 2010; 16: 407-416.

11. Lewis BP, Burge CB, Bartel DP. Conserved seed pairing, often flanked by adenosines, indicates that thousands of human genes are microRNA targets. Cell. 2005; 120: 15-20.

12. Bartel DP. MicroRNAs: genomics, biogenesis, mechanism, and function. Cell. 2004; 116: 281-297.

13. Guo H, Ingolia NT, Weissman JS, et al. Mammalian microRNAs predominantly act to decrease target mRNA levels. Nature. 2010; 466: 835-840.

14. Sayed D, Abdellatif M. MicroRNAs in development and disease. Physiol Rev. 2011; 91: 827-887.

15. Li X. MiR-375, a microRNA related to diabetes. Gene. 2014; 533: 1-4.

16. Guay C, Roggli E, Nesca V, et al. Diabetes mellitus, a microRNA-related disease? Transl Res. 2011; 157: 253-264.

17. Fernandez-Valverde SL, Taft RJ, Mattick JS. MicroRNAs in beta-cell biology, insulin resistance, diabetes and its complications. Diabetes. 2011:60: 1825-1831.

18. Heneghan HM, Miller N, Kerin MJ. Role of microRNAs in obesity and the metabolic syndrome. Obes Rev. 2010; 11: 354-361.

19. Muhonen P, Holthofer H. Epigenetic and microRNA-mediated regulation in diabetes. Nephrol Dial Transplant. 2009; 24: 1088-1096.

20. Baroukh N, Ravier MA, Loder MK, et al. MicroRNA-124a regulates Foxa2 expression and intracellular signaling in pancreatic beta-cell lines. J Biol Chem. 2007; 282: 19575-19588.

21. Wang HJ, Huang YL, Shih YY, et al. MicroRNA-146a Decreases High Glucose/Thrombin-Induced Endothelial Inflammation by Inhibiting NAPDH Oxidase 4 Expression. Mediators Inflamm. 2014; 2014: 379537.

22. Ciccacci C, Di Fusco D, Cacciotti $L$, et al. MicroRNA genetic variations: association with type 2 diabetes. Acta Diabetol. 2013; 50: 867-872

23. Sole X, Guino E, Valls J, et al. SNPStats: a web tool for the analysis of association studies. Bioinformatics. 2006; 22: 1928-1929.

24. Chan JC, Malik V, Jia W, et al. Diabetes in Asia: epidemiology, risk factors, and pathophysiology. JAMA. 2009; 301: 2129-2140.

25. Yoon $\mathrm{KH}$, Lee JH, Kim JW, et al. Epidemic obesity and type 2 diabetes in Asia. Lancet. 2006; 368: 1681-1688.

26. $\mathrm{Yu} \mathrm{W}, \mathrm{Hu} \mathrm{C}$, Jia W. Genetic advances of type 2 diabetes in Chinese populations. J Diabetes. 2012; 4: 213-220.

27. Baroukh NN, Van Obberghen E. Function of microRNA-375 and microRNA-124a in pancreas and brain. FEBS J. 2009; 276: 6509-6521.

28. Sebastiani G, Po A, Miele E, et al. MicroRNA-124a is hyperexpressed in type 2 diabetic human pancreatic islets and negatively regulates insulin secretion. Acta Diabetol. 2014

29. Qi L, Hu Y, Zhan Y, et al. A SNP site in pri-miR-124 changes mature miR-124 expression but no contribution to Alzheimer's disease in a Mongolian population. Neurosci Lett. 2012; 515: 1-6.

30. Kim SY, Kim AY, Lee HW, et al. miR-27a is a negative regulator of adipocyte differentiation via suppressing PPARgamma expression. Biochem Biophys Res Commun. 2010; 392: 323-328.

31. Sun $\mathrm{Q}, \mathrm{Gu} \mathrm{H}$, Zeng $\mathrm{Y}$, et al. Hsa-mir-27a genetic variant contributes to gastric cancer susceptibility through affecting miR-27a and target gene expression. Cancer Sci. 2010; 101: 2241-2247.

32. Ciccacci C, Morganti R, Di Fusco D, et al. Common polymorphisms in MIR146a, MIR128a and MIR27a genes contribute to neuropathy susceptibility in type 2 diabetes. Acta Diabetol. 2014; 51: 663-671.

33. Bhaumik D, Scott GK, Schokrpur S, et al. Expression of microRNA-146 suppresses NF-kappaB activity with reduction of metastatic potential in breast cancer cells. Oncogene. 2008; 27: 5643-5647.

34. Patel S, Santani D. Role of NF-kappa B in the pathogenesis of diabetes and its associated complications. Pharmacol Rep. 2009; 61: 595-603.

35. Ndisang JF. Role of heme oxygenase in inflammation, insulin-signalling, diabetes and obesity. Mediators Inflamm. 2010; 2010: 359732

36. Balasubramanyam $M$, Aravind $S$, Gokulakrishnan $\mathrm{K}$, et al. Impaired miR-146a expression links subclinical inflammation and insulin resistance in Type 2 diabetes. Mol Cell Biochem. 2011; 351: 197-205.

37. Feng B, Chen S, McArthur K, et al. miR-146a-Mediated extracellular matrix protein production in chronic diabetes complications. Diabetes. 2011; 60: 2975-2984
38. $\mathrm{Xu} \mathrm{J}, \mathrm{Wu} \mathrm{W}$, Zhang $\mathrm{L}$, et al. The role of microRNA-146a in the pathogenesis of the diabetic wound-healing impairment: correction with mesenchymal stem cell treatment. Diabetes. 2012; 61: 2906-2912.

39. Jazdzewski K, Murray EL, Franssila K, et al. Common SNP in pre-miR-146a decreases mature miR expression and predisposes to papillary thyroid carcinoma. Proc Natl Acad Sci U S A. 2008; 105: 7269-7274.

40. Kong L, Zhu J, Han W, et al. Significance of serum microRNAs in pre-diabetes and newly diagnosed type 2 diabetes: a clinical study. Acta Diabetol. 2011; 48: 61-69.

41. Rong Y, Bao W, Shan Z, et al. Increased microRNA-146a levels in plasma of patients with newly diagnosed type 2 diabetes mellitus. PLoS One. 2013; 8: e73272.

42. Zampetaki A, Kiechl S, Drozdov I, et al. Plasma microRNA profiling reveals loss of endothelial miR-126 and other microRNAs in type 2 diabetes. Circ Res. 2010; 107: 810-817.

43. Kantharidis P, Wang B, Carew RM, et al. Diabetes complications: the microRNA perspective. Diabetes. 2011; 60: 1832-1837. 